\section{Some puzzling results on the Poggendorff illusion*}

\section{A. W. PRESSEY, University of Manitoba, Winnipeg, Manitoba R3T 2N2, Canada and \\ O. SWEENEY, Mount Allison University, Sackville, New Brunswick, Canada}

Results obtained by Weintraub and Krantz on the Poggendorff illusion were critically evaluated. It was also shown that the assimilation theory of the Poggendorff illusion adequately predicted the form of the function that results from rotating one of the oblique arms.

A recent analysis of the Poggendorff configuration reduces that illusion to assimilative processes akin to those that occur in the Mueller-Lyer figure (Pressey, 1971). Basically, it is argued that, in a simplified target such as that shown in Fig. 1A, $O$ projects a series of obliques as shown in Fig. 1B, only one of which coincides with the veridical projection (the standard magnitude). It is also argued that, because of differential attention, the shorter obliques are weighted more heavily than the longer obliques, with the result that the standard magnitude assimilates and appears shorter than it really is. Thus, when $O$ chooses a projection from this series, he chooses a line that is shorter than the standard, with the result that the oblique appears to point above the point of objective continuation. ${ }^{1}$

In 1971, Weintraub and Krantz reported data which showed that, in an impoverished version almost identical to Fig. 1A, no illusion was exhibited. It would seem that their study provides a simple and conclusive disproof of the assimilation theory of the Poggendorff illusion. The problem is that their finding is contradicted by several studies that have been reported in the literature. In 1956, Kobayashi used a figure similar to $1 \mathrm{~A}$ in different orientations, with different lengths of oblique line, different gaps between oblique and main line, and different angles of oblique. In every one of his 20 conditions, he found an illusion in the same direction as that produced by the classical configuration. In 1969, Pressey and Sweeney found a large and highly reliable illusion with the impoverished version. In addition, they found that (1) the impoverished version correlated highly with the full version of the illusion and that (2) successive trials effects were almost identical in the two types of targets. In 1970, Pressey and Dewar measured the

*This research was supported by the National Research Council of Canada (AO-177). Reprints may be obtained from the Department of Psychology, University of Manitoba, Winnipeg, Manitoba R 3T 2N2. Canada. illusion in the impoverished target in 72 Os and found that 66 of them exhibited a positive illusion. In addition, the impoverished versio.1 correlated negatively with a modified Poggendorff target which produced a reversed illusion much in the way the ingoing form of the Mueller-Lyer illusion correlates negatively with the outgoing form (Christie, 1969; Smith, 1906). Finally, in a recent study (Pressey \& Sweeney, 1972), 56 of 64 Os exhibited a positive illusion on the impoverished target. With such large proportions of Os showing a positive illusion on the impoverished target, together with the fact that the impoverished illusion appears to behave lawfully, it is difficult to believe that the data obtained by Weintraub and Krantz are valid.

Weintraub and Krantz also measured the illusion produced by a target similar to that shown in Fig. 2 and found no significant effect. Now, it has been shown by Green and Hoyle (1964) and by Restle (1969) that a configuration in which the acute angles alone are present produces a reversed illusion. But Fig. 2 is an intermediate stage between Fig. 1 (no acute angles) and the target used by Restle (two acute angles) in the sense that one acute angle is present. If it is true that Fig. $1 \mathrm{~A}$ produces no illusion, Fig. 2 should have produced a reversed illusion to be consistent with previous data. In the study by Pressey and Sweeney (1972), measures of illusion were obtained on a target similar to Fig. 2. It was found that a large positive illusion was present, but this illusion was significantly smaller than that produced by a target like 1A. In other words, the presence of one acute angle does reduce the illusion. Thus, whereas the results obtained in our laboratory are consistent with what would be expected on the basis of previous data, those obtained by Weintraub and Krantz are not.

A third puzzling result obtained by Weintraub and Krantz is in reference to the magnitude of illusion as a function of distance between parallel lines. They concluded that the illusion is an increasing function of distance. However, such a conclusion could be misleading, because, strictly speaking, Weintraub and Krantz and other investigators, such as Velinsky (1925), have only shown that their particular measure of illusion increases as a function of distance. Their measure consisted of the difference between the POE and the PSE along one of the parallel lines. But it is also possible to measure the illusion in terms of angular distortion, as shown in Fig. 3.
A

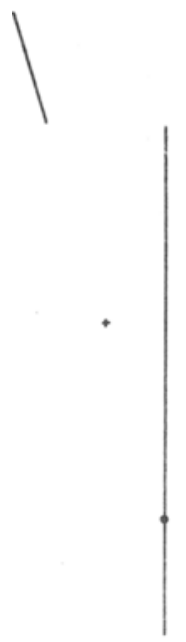

Fig. 1. (A) An impoverished version of the Poggendorff illusion. (B) Hypothetical projections of the oblique on the left toward the vertical line. The arcs represent hypothetical attentive fields which vary in size. 


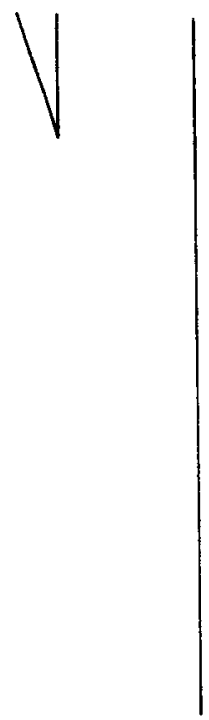

Fig. 2. A variation of the Poggendorff illusion.

Line a would be the objective continuation of the given oblique, and the intersection of $b$ and $c$ would be the PSE. The dependent variable would then be the angle formed by Lines $a$ and $b$. It should be clear that one can increase the distance between parallel lines and obtain a linearly increasing illusion (as measured by $c$ in Fig. 3) despite the fact that the angle formed $b y a$ and $b$ remains unchanged. The increase in $c$ would simply reflect the fact that the distance between two intersecting lines increases as the distance from the origin increases. Thus, a critical question that remains is whether distance between parallels affects illusion as measured by angular distortion - a measure which appears to be just as valid as linear distortion.

The data obtained by Weintraub and Krantz with the two most acute angles were transformed into angular distortion. The transformed results shcwed that there was a consistent decrease in illusion from the $7.5-\mathrm{mm}$ width to the $30-\mathrm{mm}$ width and then a slight reversal at the $60-\mathrm{mm}$ width. It is not possible to assess this trend for statistical significance, and one would be tempted to dismiss it as an unreliable one were it not for the fact that it is an exact reversal of the trend obtained in our laboratory.

\section{METHOD}

The Ss were 34 men and 73 women enrolled in an introductory psychology course at the University of Manitoba. Only individuals who had good vision, with or without glasses, were asked to participate.

The materials consisted of a practice and an experimental booklet made up of $28 \times 21.7 \mathrm{~cm}$ sheets of paper. The experimental booklet consisted of five different targets which varied in distance between parallel lines. These distances were $5,10,20,30$, and $40 \mathrm{~mm}$. Each booklet contained a different random order of distances. All targets were black lines, $0.75 \mathrm{~mm}$ thick, drawn on white paper. The two parallel lines were $195 \mathrm{~mm}$ long. The oblique line was $40 \mathrm{~mm}$ long and formed a $25 \cdot \mathrm{deg}$ angle $35 \mathrm{~mm}$ from the top of the left parallel line. The oblique on the bottom right was omitted. Each target was preceded by a sheet of heavy blue paper, which prevented $\mathbf{S}$ from seeing the target that was to follow.

The practice booklets were similar to the experimental booklets, except that only two targets were used. The configuration consisted of parallel lines $200 \mathrm{~mm}$ long and $25 \mathrm{~mm}$ apart. The oblique line was $40 \mathrm{~mm}$ long and formed a 70-deg angle $160 \mathrm{~mm}$ from the top of the left parallel line.

The Ss, who were seated two to a table separated by a wooden screen, placed their preferred hand on a white spot that was located at the edge of the table just in front of them. The $\mathbf{E}$ explained that on the signal "turn," $S$ was to turn the first blue page and inspect the target for $10 \mathrm{sec}$. On the signal "now," he was to respond by marking a fine, but clear, dot on the right vertical line at the point where the oblique line would join if it were extended. The target page was then turned, and $S$ was to wait for the next signal.

After $S$ responded on the practice targets, the booklets were collected and checked to ensure that the procedure was understood. Only two Ss did not understand the initial instructions, and a quick explanation

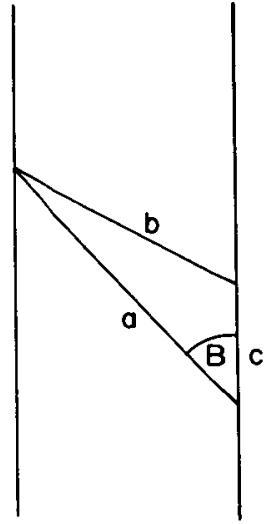

Fig. 3. Relationship between the objective transversal (a) and the subjective transversal (b) in the Poggendorff illusion. See text for explanation.

rectified the problem. The Ss were then tested on the experimental targets under the procedure described above. The instructions also specified that only one choice could be made on each target and that arm movements to project the oblique were not allowed. One $S$ did use arm movements, and his data were not used.

\section{RESULTS}

The angular deviation from the POE was calculated for each target. These scores were subjected to an analysis of variance for repeated measures. The $F$ ratio for the main effect of distance was $3.90(\mathrm{df}=4,424 ; p<.01)$. The results are shown in Fig. 4. The decrement in illusion from the $30-\mathrm{mm}$ distance to the $40-\mathrm{mm}$ distance was not significant when evaluated by

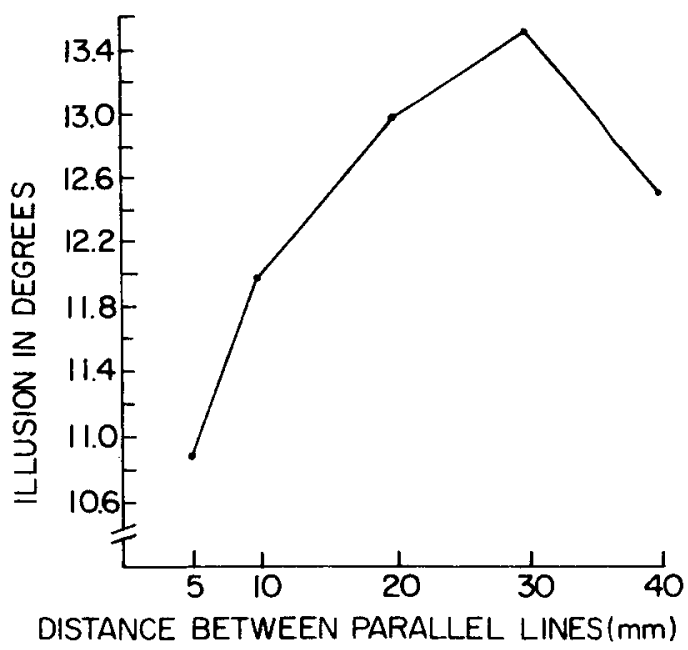

Fig. 4. The Poggendorff illusion (angular distortion) as a function of distance between parallel lines. 


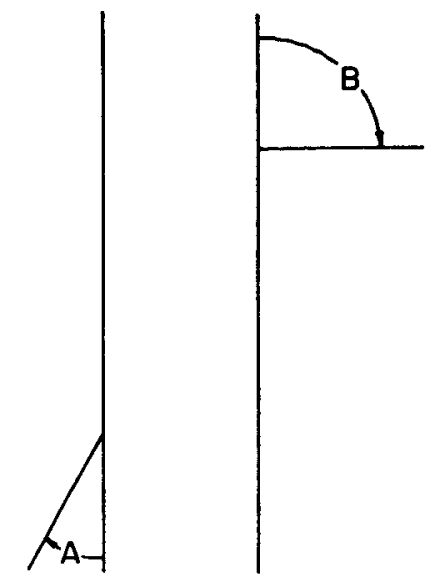

Fig. 5. Variation of the Poggendorff target employed by Weintraub and Krantz (1971).

\section{Scheffé's test $(\mathbf{p}>.10)$.}

The results clearly indicate that the illusion, as measured by angular distortion, increases as the distance between parallel lines increases, and this is the exact opposite of the decreasing trend found by Weintraub and Krantz. The only reasonable conclusion at this point seems to be that the nature of the function relating illusion (as measured by angular distortion) to distance between parallel lines is unresolved. It is possible that the different results are due to different psychophysical methods or to different positions in which the variable stimulus is located on the Poggendorff targets. At the present time, neither of these factors appears to have great theoretical significance, but, as theories of geometric illusions become more sophisticated, the relevance of the different trends may become clear.

Finally, one variation of the Poggendorff target employed by Weintraub and Krantz is interesting from the viewpoint of assimilation theory. They measured the illusion with the lower left oblique and varied the angle, $B$, from 0 to $129.8 \mathrm{deg}$ in the direction of the arrow (see Fig. 5). Their results showed that as Angle B increased, the illusion decreased at first and then increased until, at $129.8 \mathrm{deg}$, it was larger than the illusion at $B=0$ deg. They wrote, "We are convinced that the effects of $B$, though small ... are real. At present we have no further interpretation of the form of the functions... [p. 259]." However, their results are readily explained on the basis of assimilation theory.

Consider Fig. 1B, in which there are projections which are both shorter and longer than the standard magnitude. If the shorter magnitudes are of primary importance, the illusion will be in the classical direction. On the other hand, if longer magnitudes are emphasized (as in the case when we amputate the target until only the acute angles remain), a reverse illusion will occur (Pressey, 1971). Now consider a more complicated version of Fig. 1A, in which an oblique $X$ is added at an angle of $8 \mathrm{deg}$ (Fig. 6). The addition of $X$ creates the possibility that obliques will be projected against it. These obliques will be longer than the standard magnitude, and a reversed illusion should result. This reversed illusion will subtract from the classical illusion and produce a smaller illusion than would result if $X$ were eliminated. Now imagine that $X$ is rotated until it forms an angle of about $130 \mathrm{deg}$. The projections that it would trigger would all be shorter than the standard magnitude. Of course, the illusion should be positive, and, if it is added to the illusion produced by the vertical line, the total should be greater than the case in which $X$ was eliminated. Thus, if $X$ were systematically rotated from 0 to $129.8 \mathrm{deg}$, the illusion would decrease at first and then begin to increase until, at $129.8 \mathrm{deg}$, it was larger than the case in which $\mathrm{X}=0 \mathrm{deg}$. This is precisely the type of function obtained by Weintraub and Krantz.

In order to determine the quantitative function which would result from rotating $X$, the formula used to predict the Ponzo illusion (Pressey, Butchard, \& Scrivner, 1971) was employed. The formula is as follows :

$$
I=\frac{\sum_{g=1}^{N}\left(1-\frac{D c m}{D_{p}}\right)\left(L_{c m}-L_{s m}\right)}{N}
$$

where $L_{c m}=$ length of contextual magnitude (see text), $L_{s m}=$ length of standard magnitude (see text), Dcm = distance from the center of the attentive field to the most distant point of the contextual magnitude, $D_{p}$ $=$ distance from the center of the attentive field to the periphery, and $N$ $=$ all contextual magnitudes that are sampled and that fall entirely within the attentive field.

The target chosen for analysis was similar to Fig. 1B, in which the oblique on the left formed an angle of $16.7 \mathrm{deg}$ with the vertical axis. The vertical line was $13 \mathrm{~cm}$ long and was located in such a fashion that the oblique pointed to a position $10 \mathrm{~cm}$ below the top of that line. The horizontal distance from the bottom of the oblique to the vertical line was $30 \mathrm{~mm}$.

The vertical line was marked every $5 \mathrm{~mm}$, and a line was drawn from the bottom of the oblique to each of these marks. This resulted in 27 hypothetical projections or contextual magnitudes, 20 of which were shorter than the standard magnitude and 6 of which were longer than the standard magnitude. The second oblique, corresponding to $X$ in Fig. 6, was $3 \mathrm{~cm}$ long and was located at 16.7, 31.0, $50.2,67.4,90.0$, and $129.8 \mathrm{deg}$ from the vertical. It, too, was marked every $5 \mathrm{~mm}$, and projections were drawn from the marks to the bottom of the oblique on the left. The illusion was calculated separately for the vertical line and for the rotated oblique, and the scores were added to obtain the predicted value.

The center of the attentive field was assumed to be in the middle of the standard magnitude (see cross in Fig. 1A), and, in calculating the function, four attentive fields were employed. The radii of these fields were set at $80,100,120$, and $140 \mathrm{~mm}$. The predicted amount of shrinkage of the standard magnitude as a function of angle of $\mathrm{X}$ and size of attentive field is shown in Fig. 7. Clearly, the functions are of the "U" type, in which the illusion at $X=129.8 \mathrm{deg}$ is greater than the illusion at $\mathrm{X}=0 \mathrm{deg}$.

It might be argued that the predicted shrinkage of the standard magnitude (i.e., the transversal between the parallel lines) does not correspond to anything like that which we measure in the Poggendorff illusion. Normally, linear displacement from the POE along the parallel line is employed as a measure of illusion. This is a valid argument, and it appears

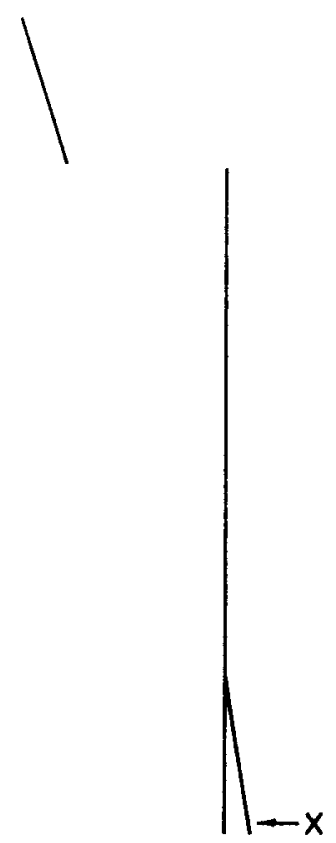

Fig. 6. A modified Poggendorff illusion. See text for explanation. 


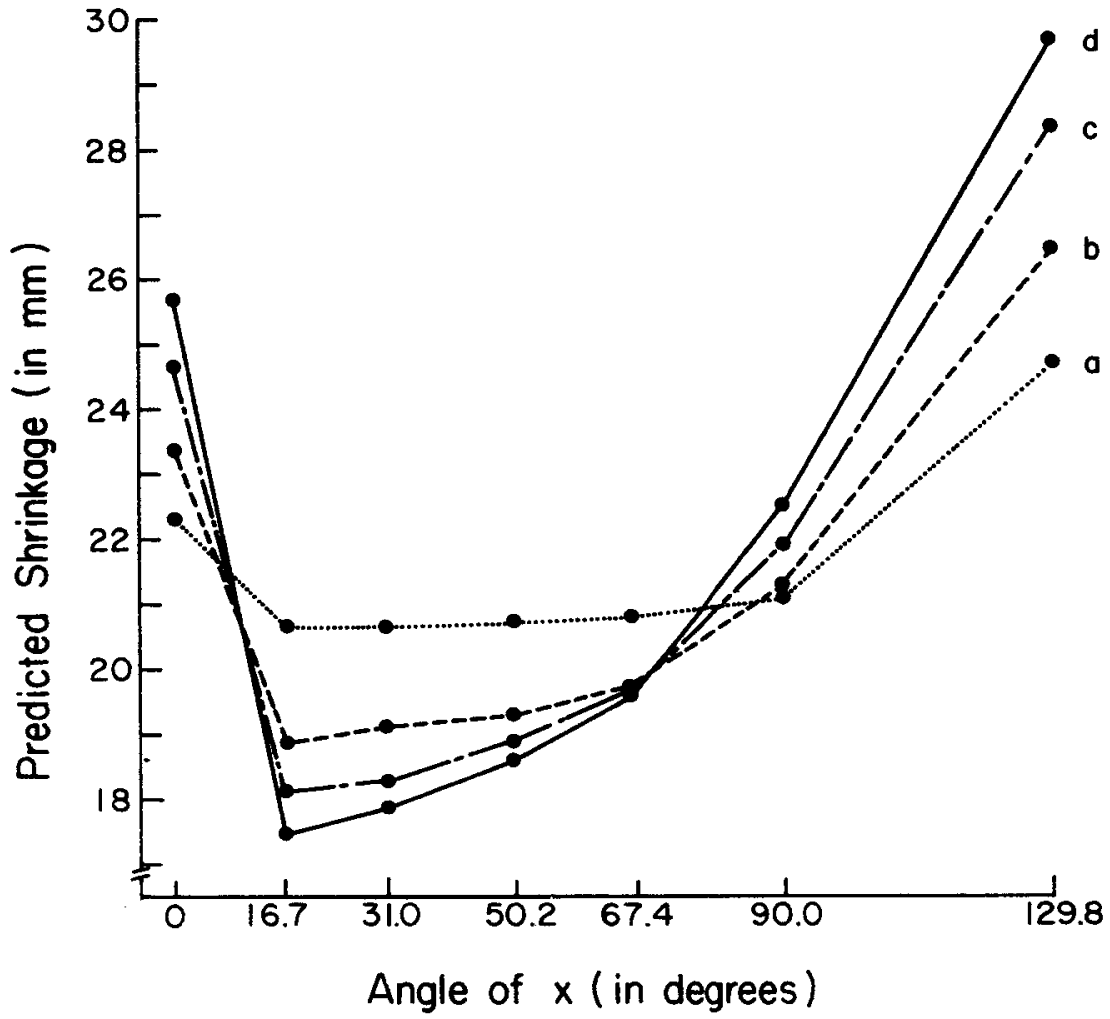

Fig. 7. Predicted shrinkage of standard magnitude as a function of rotated oblique (X) and attentive fields $(a, b, c$, and $d)$.

that a transformation of the predicted score is necessary.

Consider Fig. 3, in which the transversal a is the standard magnitude. Now, since a shrinks, it appears shorter and its apparent length can correspond to $b$ in Fig. 3 . The value of $b$ is equal to a minus the amount of shrinkage predicted by Formula 1. It is obvious that abc forms a triangle in which c corresponds to the linear deviation that is normally measured in the Poggendorff illusion. And, since $a$ is given and Angle $B$ is given, and $b$ is calculated, the derivation of the value of c follows from the law of sines.

The conversion of shrinkage scores into scores of linear deviation was carried out for the function shown in Fig. 7 corresponding to an attentive field of $100 \mathrm{~mm}$. The results are shown in Fig. 8. Also shown in Fig. 8 are the results obtained by Weintraub and Krantz. The shapes of the two curves are, of course, very similar, but what is more striking is that the formula predicts the absolute magnitude of illusion fairly well. It should be noted that a better fit could have been obtained had the function corresponding to an attentive field of $140 \mathrm{~mm}$ been employed. But there was a reason for using the $100-\mathrm{mm}$ field. In the Ponzo illusion, an attentive field subtending a visual corresponds to $18.8 \mathrm{deg}$ of visual angle (viewing distance equal to $61 \mathrm{~cm}$ ). Thus, if we employ Formula 1 to predict a Poggendorff illusion in which the angle of the oblique and the distance between parallel lines are equal to those employed by Weintraub and Krantz, and if we employ an attentive field similar to the one used to predict the Ponzo illusion, then both the shape of the function and the absolute level of the Poggendorff illusion are predicted reasonably well.

One aspect of the results shown in Fig. 8 deserves special emphasis. When the rotated oblique $X$ is at $16.7 \mathrm{deg}$ (and thus directly in line with the oblique on the left), the predicted and obtained illusion is smaller than the case in which $X$ is eliminated. Also, Weintraub and Krantz employed a control target in which the parallels were omitted and only the obliques remained. This control target produced a negative illusion. Now, the interesting thing is that such a target is the limiting case of the outgoing form of the Mueller-Lyer illusion, and, as such, the standard length between the obliques should appear to elongate. In their empirical study, Smith and Sowton (1907) concluded that "... the presence of a line at the end of a standard line tends to increase the apparent length of the standard line ... [p. 205]." But, according to assimilation theory, elongation of the standard magnitude is associated with a negative Poggendorff illusion; thus, these particular results obtained by

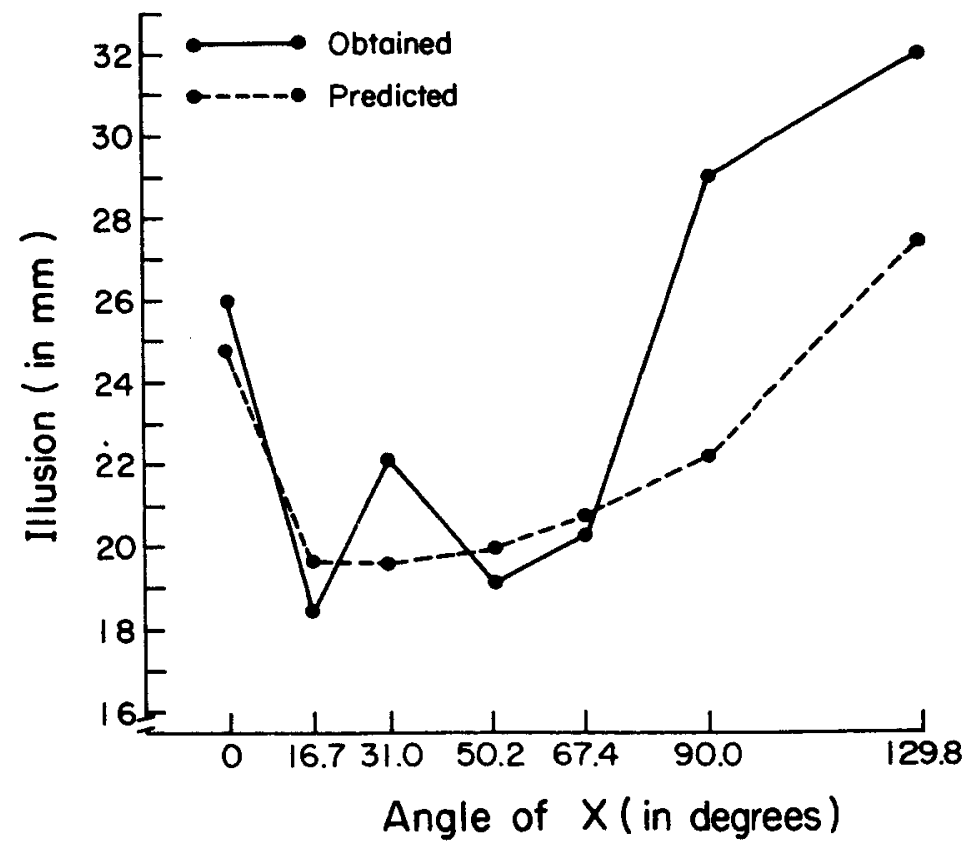

Fig. 8. Predicted and obtained Poggendorff illusion as a function of angle of rotated oblique. The obtained data are from Weintraub \& Krantz (1971). 
Weintraub and Krantz make sense.

There is one additional way in which the results obtained by Weintraub and Krantz (shown in Fig. 8) bear on the assimilation theory of the Poggendorff illusion. If it is granted that an $O$ projects a series of obliques as shown in Fig. 1B, there are two ways of interpreting what happens next. The first interpretation is that the important aspect is the orientation of the obliques so that there is assimilation to an average orientation. The second point of view is that it is the length of the obliques which is important, and this, of course, is what has been maintained since the assimilation theory was first applied to the Poggendorff illusion (Pressey, 1971). So far, there have not been any good grounds on which a choice between the two alternatives could be made. Certainly, the second interpretation is less parsimonious, since it must be assumed that length is somehow translated into orientation. Nevertheless, the data of Weintraub and Krantz support the second, or length, interpretation. If it were simply orientation of obliques, the only portion of the curve in Fig. 8 that would be lower than the base level would be at the angles between 0 and $16.7 \mathrm{deg}$. At $16.7 \mathrm{deg}$, the illusion should be equal to that obtained at $0 \mathrm{deg}$. Then, as $\mathrm{X}$ was rotated beyond $16.7 \mathrm{deg}$, the illusion should always be larger than the base level. Clearly, this is not the kind of function that Weintraub and Krantz obtained, and, therefore, the orientation interpretation is not tenable.

\section{REFERENCES}

CHRISTIE, B. P. A contaminating factor in the measurement of geometric illusions. Psychonomic Science, 1969, 17, 69-70.

GREEN, R. T., \& HOYLE, E. M. The influence of spatial orientation on the Poggendorff illusion. Acta Psychologica, 1964, 22, 348-366.

KOBAYASHI, T. Analytical study of displacement in visual perception (I). Japanese Psychological Research, 1956, 3. 37-44.

PRESSEY, A. W. An extension of assimilation theory to illusions of size, area, and direction. Perception \& area, and direction, Percep

PRESSEY, A. W., BUTCHARD, N., \& SCRIVNER, L. Assimilation theory and the Ponzo illusion: Quantitative predictions. Canadian Journal of Psychology, 1971, 25, 486-497.

PRESSEY, A. W., \& DEWAR, M. J. A reversed Poggendorf illusion. Psychonomic Science, 1970, 20, 227-228.

PRESSEY, A. W., \& SWEENEY, 0. A variation of the Poggendorff illusion. Perceptual \& Motor Skills, 1969, 28, 883-886.

PRESSEY, A. W., \& SWEENEY, O. Acute angles and the Poggendorff illusion. Quarterly Journal of Experimental Psychology, 1972, 24, 169-174.

RESTLE F Illusions of bent line Perception \& Psychophysies, 1969, 5, 273-274.

SMITH, W. G. A study of some correlations of the Muller-Lyer visual illusions and allied phenomena. British Journal of Psychology, 1906, 2, 16-51.

SMITH, w. G., \& SOWTON, s. C. M. Observations on spatial contrast and confluence in visual perception. British Journal of Psychology, 1907, 2, 196-219.

VELINSKY, S. Explication physiologique de l'illusion de Poggendorff. Année Psychologique, 1925, 26, 107-116.

WEINTRAUB, D. J \& KRANTZ, D. $H$. The Poggendorff illusion: Amputations, rotations, and other perturbations. Perception \& Psychophysics, 1971, 10, 257-264.

\section{NOTE}

1. The exact process by means of which apparent length is translated into apparent orientation is not clear, and all that is intended at this stage is that there is a direct relationship between direction and amount of change of the standard magnitude and direction and amount of Poggendorff illusion.

(Received for publication March 6, 1972; revision received June 27,1972 .) 